\title{
BASIC SEQUENCES IN $F$-SPACES AND THEIR APPLICATIONS
}

\author{
by N. J. KALTON \\ (Received 30th June 1973)
}

\section{Introduction}

The aim of this paper is to establish a conjecture of Shapiro (10) that an $F$-space (complete metric linear space) with the Hahn-Banach Extension Property is locally convex. This result was proved by Shapiro for $F$-spaces with Schauder bases; other similar results have been obtained by Ribe (8). The method used in this paper is to establish the existence of basic sequences in most $F$-spaces.

It was originally stated by Banach that every $B$-space contains a basic sequence, and proofs have been given by Bessaga and Pelczynski (1), (2), Gelbaum (4) and Day (3). In (1) Bessaga and Pelczynski give a general method of construction in locally convex $F$-spaces, but we shall show in Section 3 that this construction can be modified to apply in any $F$-space $(X, \tau)$ on which there is a weaker vector topology $\rho$ such that $\tau$ has a base of $\rho$-closed neighbourhoods. The basic result of the paper is Theorem 3.2, and this is a natural generalisation of a locally convex version due to Bessaga and Mazur and given (essentially) in Pelczynski (6), (7).

In Section 4 we study the problem of existence of a basic sequence in an arbitrary $F$-space, and show that in fact repeated applications of Theorem 3.2 give a basic sequence in any $F$-space with a non-minimal topology. Since the only example we know of a minimal $F$-space is the space $\omega$ of all sequences (which has a basis) it seems likely that every $F$-space contains a basic sequence.

The results of Section 5 do not depend on Section 4; in this section are gathered together the applications of the existence theory of Section 3. We show that if $(X, \tau)$ is an $F$-space and $\rho \leqq \tau$ is a topology defining the same closed linear subspaces as $\tau$, then $\rho$ and $\tau$ define the same bounded sets-a result familiar in locally convex theory. The Shapiro conjecture follows immediately. The final theorem is a generalisation of the Eberlein-Smulian theorem employing techniques developed by Pelczynski (7).

The author would like to thank Professor J. H. Shapiro for several helpful comments and supplying a copy of (8), and also the referee for pointing out some serious mistakes in the first draft of the paper. 


\section{Preliminary results}

An $F$-semi-norm $\eta$ on a vector space $X$ is a non-negative real-valued function defined on $X$ such that

(i) $\eta(x+y) \leqq \eta(x)+\eta(y)$.

(ii) $\eta(t x) \leqq \eta(x) \quad|t| \leqq 1$,

(iii) $\lim _{t \rightarrow 0} \eta(t x)=0 \quad x \in X$.

If in addition $\eta(x)=0$ implies that $x=0$ then we call $\eta$ an $F$-norm. Any vector topology on $X$ may be defined by a collection of $F$-semi-norms; any metrisable topology may be defined by one $F$-norm. From this point, unless specifically stated, all vector topologies are assumed to be Hausdorff.

Now suppose $(X, \rho)$ is a topological vector space and $\tau$ is a vector topology on $X$; we shall say that $\tau$ is $\rho$-polar if $\tau$ has a base of neighbourhoods which are $\rho$-closed.

Proposition 2.1. If $\tau$ is $\rho$-polar then $\tau$ may be defined by a collection of $F$ semi-norms $\left(\eta_{\alpha}: \alpha \in A\right)$ of the form

$$
\eta_{\alpha}(x)=\sup \left\{\lambda(x): \lambda \in \Lambda_{\alpha}\right\}
$$

where each $\Lambda_{\alpha}$ is a collection of $\rho$-continuous F-semi-norms. If $\tau$ is metrisable then $\tau$ may be defined by one such F-norm.

Proof. Let $\left(\gamma_{\alpha}: \alpha \in A\right)$ be a collection of $F$-semi-norms defining $\tau$ such that every $\tau$-neighbourhood of 0 contains a set $\left\{x: \gamma_{\alpha}(x) \leqq \varepsilon\right\}$ for some $\alpha \in A$ and $\varepsilon>0$; let $\Delta$ be the collection of all $\rho$-continuous $F$-semi-norms. We define $\Lambda_{\alpha}$ to be the collection of $F$-semi-norms of the form

$$
\lambda_{\delta}^{\alpha}(x)=\inf \left(\delta(y)+\gamma_{\alpha}(z): y+z=x\right) .
$$

(Thus $\Lambda_{\alpha}=\left\{\lambda_{\delta}^{\alpha}: \delta \in \Delta\right\}$.) As $\lambda_{\delta}^{\alpha} \leqq \delta$ each $\lambda_{\delta}^{\alpha}$ is $\rho$-continuous and an $F$-semi-norm ( $\lambda_{\delta}^{\alpha} \leqq \delta$ implies condition (iii) in particular). Now define

$$
\eta_{\alpha}(x)=\sup \left(\lambda_{\delta}^{\alpha}(x): \delta \in \Delta\right) \text {. }
$$

Clearly $\eta_{\alpha} \leqq \gamma_{\alpha}$ and so is an $F$-semi-norm. Now if $U$ is a $\tau$-neighbourhood of 0 we may find $\alpha_{1}$ and $\varepsilon>0$ such that if $x_{0} \in\left\{\overline{x: \gamma_{\alpha_{1}}(x) \leqq \varepsilon}\right\}$ (closure in $\rho$ ) then $x_{0} \in U$. Suppose now $x_{0} \in\left\{x: \eta_{\alpha_{1}}(x)<\varepsilon\right\}$; then it is easy to show that $x_{0} \in$ $\left\{\overline{x: \gamma_{\alpha_{1}}(x) \leqq \varepsilon}\right\}$ and so $\left(\eta_{\alpha}: \alpha \in A\right)$ defines $\tau$.

If $\tau$ is metrisable, $A$ may be taken to be a singleton and therefore $\tau$ may be defined by a single $F$-norm of the required type.

Proposition 2.2. Suppose $(X, \tau)$ is an $F$-space (complete metric linear space) and suppose $\rho<\tau$ is a vector topology on $X$. Then 
(i) If the net $x_{a} \rightarrow 0(\rho)$ but $x_{a} \rightarrow 0(\tau)$, then there are vector topologies $\alpha, \beta$ such that

(a) $\rho \leqq \alpha<\beta \leqq \tau$;

(b) $\beta$ is metrisable and $\alpha$-polar;

(c) $x_{a} \rightarrow 0(\alpha)$ but $x_{a} \rightarrow 0(\beta)$.

(ii) If $U$ is a $\tau$-neighbourhood of 0 but not a $\rho$-neighbourhood then there are vector topologies $\alpha, \beta$ satisfying (a), (b) and (c)' $U$ is a $\beta$-neighbourhood of 0 but not an $\alpha$-neighbourhood of 0 .

(iii) If $\tau$ is locally bounded then there is a topology $\alpha$ such that $\alpha<\tau$ but $\tau$ is $\alpha$-polar.

Proof. (i) Let $\alpha$ be the largest vector topology such that $\rho \leqq \alpha \leqq \tau$ and $x_{a} \rightarrow 0(\alpha)$ (it is easy to see that there is such a topology). Let $\beta$ be the vector topology with a base of neighbourhoods consisting of the $\alpha$-closures of $\tau$-neighbourhoods of 0 . Since $\alpha \leqq \tau$ it follows that $\alpha \leqq \beta \leqq \tau$. If $\alpha=\beta$ then the identity map $i:(X, \alpha) \rightarrow(X, \tau)$ is almost continuous and so by the Closed Graph Theorem (cf. Kelley (5), p. 213) $\alpha=\tau$ contrary to hypothesis on the net $\left(x_{a}\right)$. Therefore $\alpha<\beta$; clearly also since $\tau$ is metrisable so is $\beta$, and $x_{a} \rightarrow 0(\beta)$.

(ii) (We are grateful to J. H. Shapiro for the following simplification of the original proof.) By an application of Zorn's Lemma it may be shown that there is a maximal vector topology $\alpha$ such that $\rho \leqq \alpha \leqq \tau$ and $U$ is not an $\alpha$-neighbourhood (we do not assert that $\alpha$ is the largest such topology). Then proceed as in (i).

(iii) Follows from (ii) by considering a single bounded neighbourhood $(\beta=\tau)$.

Two vector topologies on $X$ will be called compatible if they define the same closed subspaces.

Proposition 2.3. Let $\tau$ and $\rho$ be compatible topologies on $X$; they define the same continuous linear functionals.

Proof. $f$ is $\tau$ - or $\rho$-continuous according as its null space is $\tau$ - or $\rho$-closed.

A sequence $\left(x_{n}\right)$ in a topological vector space $X$ is called a basis if every $x \in X$ has a unique expansion in the form

$$
x=\sum_{i=1}^{\infty} t_{i} x_{i}
$$

In this case we may define linear functionals $f_{n}$ such that

and linear operators $S_{n}$ by

$$
f_{n}(x)=t_{n}
$$

$$
S_{n}(x)=\sum_{i=1}^{n} t_{i} x_{i}=\sum_{i=1}^{n} f_{i}(x) x_{i} .
$$

E.M.S. $-19 / 2-\mathrm{L}$ 
If $X$ is an $F$-space then it is well known (cf. (10), (12)) that each $f_{n}$ is necessarily continuous and the family $\left\{S_{n}\right\}$ is equicontinuous.

Suppose now that $X$ is metrisable but not necessarily complete; we shall call a sequence $\left(x_{n}\right)$ in $X$ a basic sequence if it is a basis for its closed linear span in the completion of $X$. We shall call $\left(x_{n}\right)$ a semi-basic sequence if we simply have $x_{n} \notin \varlimsup \operatorname{lin}\left\{x_{n+1}, x_{n+2}, \ldots\right\}$ for every $n$.

We now give a useful and elementary criterion for a sequence $\left(x_{n}\right)$ to be basic or semi-basic. Let $\left(x_{n}\right)$ be linearly independent and let $E$ be the linear span of $\left(x_{n}\right)$; then for $x \in E$

$$
x=\sum_{i=1}^{\infty} t_{i} x_{i}
$$

uniquely where $\left(t_{i}\right)$ is finitely non-zero. Define

and

$$
f_{n}(x)=t_{n}
$$

where $S_{n}: E \rightarrow E$ is linear.

$$
S_{n} x=\sum_{i=1}^{n} f_{i}(x) x_{i}
$$

Lemma 2.4. (i) $\left(x_{n}\right)$ is semi-basic if and only if each $S_{n}$ is continuous or equivalently each $f_{n}$ is continuous.

(ii) $\left(x_{n}\right)$ is basic if and only if the family $\left\{S_{n}\right\}$ is equicontinuous.

Proof. (i) If $\left\{x_{n}\right\}$ is semi-basic, let $N_{k}$ be the null space of $f_{k}$; then $N_{k}$ is a maximal linear subspace of $E$. Then $N_{1}=\operatorname{lin}\left\{x_{i}: i \geqq 2\right\}$ and since $x_{1} \notin \bar{N}_{1}$, $N_{1}$ is closed and $f_{1}$ is continuous; while if $k \geqq 2$,

Hence

$$
N_{k}=\operatorname{lin}\left\{x_{i}: i \neq k\right\}=\operatorname{lin}\left\{x_{i}: i<k\right\}+\operatorname{lin}\left\{x_{i}: i>k\right\} .
$$

$$
\bar{N}_{k}=\operatorname{lin}\left\{x_{i}: i<k\right\}+\overline{\operatorname{lin}}\left\{x_{i}: i>k\right\},
$$

since the former space is finite-dimensional. Suppose $x_{k} \in \bar{N}_{k}$; then

$$
x_{k}=\sum_{i=1}^{k-1} t_{i} x_{i}+y
$$

where $y \in \overline{\operatorname{lin}}\left\{x_{i}: i>k\right\}$. Since $x_{k} \notin \overline{\operatorname{lin}}\left\{x_{i}: i>k\right\}$ we conclude that there is a first index $l$ such that $t_{l} \neq 0$. Then we obtain $x_{l} \in \varlimsup$ in $\left\{x_{l+1}, x_{l+2}, \ldots\right\}$ and a contradiction. Hence $x_{k} \notin \bar{N}_{k}$ and by the maximality of $N_{k}, N_{k}$ is closed and $f_{k}$ is continuous.

The converse is trivial.

(ii) (Cf. Shapiro (12), Proposition C.)

It follows from the definition of basic sequence that if $\left(x_{n}\right)$ is basic then the family $\left\{S_{n}\right\}$ is equicontinuous (consider $\left(x_{n}\right)$ as a basis of its closed linear span in the completion of $X$ ). Conversely, $S_{n}(x) \rightarrow x$ for $x \in E$ and if the family is 


\section{BASIC SEQUENCES IN $F$-SPACES}

equicontinuous $S_{n}(x) \rightarrow x$ for $x \in \bar{E}$ (closure in the completion of $X$ ), and it easily follows that $\left(x_{n}\right)$ is a basis for $\bar{E}$.

\section{Construction of basic sequences}

Lemma 3.1. Let $E$ be a finite-dimensional space and suppose $V$ is a closed balanced subset of $E$. If $V$ intersects every one-dimensional subspace of $E$ in a bounded set then $V$ is bounded.

Proof. We may suppose $E$ is normed; suppose $x_{n} \in V$ and $\left\|x_{n}\right\| \rightarrow \infty$. Then by selecting a subsequence we may suppose $\left\|x_{n}\right\|^{-1} x_{n} \rightarrow z$ where $\|z\|=1$. Then for any $N$ there is an $m$ such that for $n \geqq m,\left\|x_{n}\right\| \geqq N$ and

$$
\left\|x_{n}\right\|^{-1} x_{n} \in\left\|x_{n}\right\|^{-1} V \subset N^{-1} V \text {. }
$$

Therefore $z \in N^{-1} V$ for all $N$ and hence $V \supset \operatorname{lin}\{z\}$.

Theorem 3.2. Suppose $(X, \tau)$ is a metric linear space and $\rho$ is a vector topology on $X$ such that $\tau$ is $\rho$-polar. Suppose $\left(x_{a}\right)$ is a net such that $x_{a} \rightarrow 0(\rho)$ but $x_{a} \rightarrow 0(\tau)$; suppose $z_{1} \neq 0 \in X$. Then there is a sequence $(a(k): k \geqq 2)$ such that

$$
a(k+1)>a(k)
$$

for all $k \geqq 2$ and the sequence $\left(z_{n}\right)_{n=1}^{\infty}$ is a basic sequence where $z_{n}=x_{a(n)} n \geqq 2$.

Proof. We may suppose (Proposition 2.1) that $(X, \tau)$ is normed by an F-norm $\|\cdot\|$ such that

$$
\|x\|=\sup (\lambda(x): \lambda \in \Lambda) \text {, }
$$

where $\Lambda$ is a collection of $\rho$-continuous $F$-norms. Let $\theta>0$ be chosen such that

(i) $\left\|z_{1}\right\| \geqq 4 \theta$.

(ii) For all $a, \exists a^{\prime} \geqq a$ such that $\left\|x_{a^{\prime}}\right\| \geqq 4 \theta$.

Let $V=\{x:\|x\| \leqq \theta\}$; then $V \cap \operatorname{lin}\left\{z_{1}\right\}$ is compact (since $\left\|z_{1}\right\| \geqq 4 \theta$ ). We shall construct the sequence $(a(n): n \geqq 2)$ by induction so that if

then $E_{n} \cap V$ is compact.

$$
E_{n}=\operatorname{lin}\left(z_{1}, x_{a(2)}, \ldots, x_{a(n)}\right)
$$

Suppose $\{a(2), \ldots, a(n)\}$ have been chosen (this set can be empty at the first step, the selection of $a(2))$ and let $E_{n}=\operatorname{lin}\left(z_{1}, x_{a(2)}, \ldots, x_{a(n)}\right)$. By the inductive hypothesis $V \cap E_{n}$ is compact.

For $1 \leqq k \leqq 2^{n+3}$ let

$$
W_{k}^{n}=\left\{x:\|x\|=k \cdot 2^{-(n+3)} \theta\right\} \cap E_{n} .
$$

Each $W_{k}^{n}$ is compact and so we may choose finite subsets $U_{k}^{n}$ so that for $w \in W_{k}^{n}$ there exists $u \in U_{k}^{n}$ with

$$
\|w-u\| \leqq 2^{-(n+3)} \theta .
$$

Let $U^{n}=\bigcup_{k=1}^{2 n+3} U_{k}^{n}$, and for $u \in U^{n}$ choose $\lambda_{u} \in \Lambda$ so that

$$
\lambda_{u}(u) \geqq\|u\|-2^{-(n+3)} \theta .
$$


Then choose $b>a(n)$ so that if $c \geqq b$ then

$$
\lambda_{u}\left(x_{c}\right) \leqq 2^{-(n+3)} \theta
$$

for $u \in U^{n}$ (possible since $U^{n}$ is finite and $x_{a} \rightarrow 0(\rho)$ ).

Choose a subnet $\left(x_{d}: d \in D\right)$ of $\left(x_{c}: c \geqq b\right)$ such that $\left\|x_{d}\right\| \geqq 4 \theta$, and suppose for every such $x_{d}$ the set $V \cap$ lin $\left(E_{n}, x_{d}\right)$ is unbounded. By Lemma 3.1, for every $d$ there exists $t_{d} x_{d}+u_{d} \neq 0$ where $u_{d} \in E_{n}$ such that the linear span of $\left(t_{d} x_{d}+u_{d}\right)$ is contained in $V$. Clearly $u_{d} \neq 0$ and so we may normalize in such a way that $\left\|u_{d}\right\|=\theta$ (since $V \cap E_{n}$ is compact). Then

$$
\begin{aligned}
\left\|t_{d} x_{d}\right\| & \leqq\left\|t_{d} x_{d}+u_{d}\right\|+\left\|u_{d}\right\| \\
& \leqq 2 \theta
\end{aligned}
$$

so that $\left|t_{d}\right| \leqq 1$. Hence since $x_{d} \rightarrow 0(\rho), t_{d} x_{d} \rightarrow 0$ in $(\rho)$. By selection again of a subnet we may suppose $u_{d} \rightarrow u$ in $E_{n}$ (since $V \cap E_{n}$ is compact) and $\|u\|=\theta$. Then for any $t \in \mathbf{R}$

$$
\begin{aligned}
\|t u\| & \leqq \liminf _{d \rightarrow \infty}\left\|t\left(t_{d} x_{d}+u_{d}\right)\right\| \\
& \leqq \theta
\end{aligned}
$$

so that lin $\{u\} \subset V \cap E_{n}$, a contradiction.

Hence we may choose $a(n+1) \geqq b$ such that $\left\|x_{a(n+1)}\right\| \geqq 4 \theta$ and $V \cap E_{n+1}$ is compact. This completes the construction of $a(n)$; now let $z_{n}=x_{a(n)} n \geqq 2$. It remains to establish that by using (1) and (2) $\left(z_{n}\right)$ is a basic sequence.

For convenience we shall replace $\|\cdot\|$ by an equivalent $F$-norm $\|\cdot\|^{*}$ given by

$$
\|x\|^{*}=\min (\|x\|, \theta) \text {. }
$$

We next show that if $t_{1}, \ldots, t_{n+1}$ is a scalar sequence

$$
\left\|\sum_{i=1}^{n+1} t_{i} z_{i}\right\|^{*} \geqq\left\|\sum_{i=1}^{n} t_{i} z_{i}\right\|^{*}-2^{-(n+1)} \theta .
$$

Choose the greatest integer $k$ such that

$$
\left\|\sum_{i=1}^{n} t_{i} z_{i}\right\|^{*} \geqq k \cdot 2^{-(n+3)} \theta .
$$

Then $0 \leqq k \leqq 2^{n+3}$; if $k=0$ there is nothing to prove. If $k \geqq 1$ then we may choose a scalar $s$ with $|s| \leqq 1$ such that

Then choose $u \in U_{k}^{n}$ so that

$$
\left\|\sum_{i=1}^{n} s t_{i} z_{i}\right\|=k \cdot 2^{-(n+3)} \theta .
$$

$$
\left\|u-\sum_{i=1}^{n} s t_{i} z_{i}\right\| \leqq 2^{-(n+3)} \theta .
$$


If $\left|s t_{n+1}\right| \leqq 1$ then

$$
\begin{aligned}
\left\|u+s t_{n+1} z_{n+1}\right\| & \geqq \lambda_{u}(u)-\lambda_{u}\left(z_{n+1}\right) \\
& \geqq(k-2) \cdot 2^{-(n+3)} \theta
\end{aligned}
$$

by (1) and (2). If $\left|s t_{n+1}\right| \geqq 1$ then

$$
\begin{aligned}
\left\|u+s t_{n+1} z_{n+1}\right\| & \geqq\left\|z_{n+1}\right\|-\|u\| \\
& \geqq 3 \theta \geqq(k-2) 2^{-(n+3)} \theta .
\end{aligned}
$$

Hence

$$
\begin{aligned}
\left\|s \sum_{i=1}^{n+1} t_{i} z_{i}\right\| & \geqq(k-2) 2^{-(n+3)} \theta-2^{-(n+3)} \theta \\
& =(k-3) 2^{-(n+3)} \theta \\
& \geqq\left\|\sum_{i=1}^{n} t_{i} z_{i}\right\|^{*}-2^{-(n+1)} \theta .
\end{aligned}
$$

Hence since $|s| \leqq 1$

and (3) follows.

$$
\left\|\sum_{i=1}^{n+1} t_{i} z_{i}\right\| \geqq\left\|\sum_{i=1}^{n} t_{i} z_{i}\right\|^{*}-2^{-(n+1)} \theta
$$

From (3) it is clear that $\left(z_{n}\right)$ is linearly independent for if $\left\|\sum_{i=1}^{n} t_{i} z_{i}\right\| \geqq \theta$ then $\left\|\sum_{i=1}^{n+1} t_{i} z_{i}\right\| \geqq \frac{1}{2} \theta ;$ thus if $\sum_{i=1}^{n+1} t_{i} z_{i}=0$, then for every $s,\left\|s \sum_{i=1}^{n} t_{i} z_{i}\right\| \leqq \theta$ and so since $V \cap E_{n}$ is compact, $\sum_{i=1}^{n} t_{i} z_{i}=0$. Let $E$ be the linear span of $\left\{z_{n}\right\}$ and define $S_{k}$ by

$$
S_{k}\left(\sum_{i=1}^{\infty} t_{i} z_{i}\right)=\sum_{i=1}^{k} t_{i} z_{i}
$$

where $\left(t_{i}\right)$ is finitely non-zero. Then by (3)

$$
\left\|S_{n+k} x\right\|^{*} \geqq\left\|S_{n} x\right\|^{*}-2^{-n} \theta \quad(k \geqq 0)
$$

and therefore for $x \in E$ and $n \geqq 1$

$$
\|x\|^{*} \geqq\left\|S_{n} x\right\|^{*}-2^{-n} \theta .
$$

Suppose $\left\|x_{m}\right\| \rightarrow 0$ but $\left\|S_{k} x_{m}\right\| \rightarrow 0$; then since $V \cap E_{k}$ is compact we may, by selecting a subsequence and multiplying by a bounded sequence of scalars, suppose that $\left\|S_{k} x_{m}\right\|=\theta$. Thus $\left\|x_{m}\right\| \geqq \frac{1}{2} \theta>0$, and we have a contradiction. Thus each $S_{k}$ is continuous.

To establish equicontinuity of $\left\{S_{m}: m \geqq 1\right\}$ we must show that if $p(m)$ is any sequence and $x_{m} \rightarrow 0$ then $S_{p(m)} x_{m} \rightarrow 0$. Suppose not; then we may suppose

for all $m$. Then

$$
\left\|S_{p(m)} x_{m}\right\|^{*} \geqq \gamma>0
$$

$$
\left\|x_{m}\right\|^{*} \geqq \gamma-2^{-p(m)} \theta
$$


and as $\left\|x_{m}\right\|^{*} \rightarrow 0$ we conclude that $p(m)$ is bounded. But then we may select a constant subsequence and this contradicts the continuity of each $S_{n}$. Thus by Lemma 2.4 we have established the theorem.

Corollary 3.3. Under the assumptions of Theorem 3.2 suppose $\mu$ is a pseudometrisable topology on $X$ such that $\mu \leqq \rho$. Then $\left(z_{n}\right)$ may be chosen so that $z_{n} \rightarrow 0(\mu)$.

An examination of the proof of Theorem 3.2 reveals that we can insist that $\eta\left(z_{n}\right) \rightarrow 0$ for any single $\rho$-continuous $F$-semi-norm.

Corollary 3.4. Suppose that $(X, \tau)$ is an F-space and that $\rho$ is a vector topology on $X$ with $\rho<\tau$. Suppose $x_{a} \rightarrow 0(\rho)$ but $x_{a} \rightarrow 0(\tau)$, and that $z_{1} \in X$. Then there is a sequence $a(k)$ so that $a(k+1)>a(k) k \geqq 2$ and such that the sequence $\left(z_{n}\right)$ is a semi-basic sequence where $z_{n}=x_{a(n)} n \geqq 2$.

Proof. Proposition 2.2 combined with Theorem 3.2 establishes that we may choose $\left(z_{n}\right)$ to be a basic sequence in a weaker topology than $\tau$. This clearly implies that $\left(z_{n}\right)$ is at least a semi-basic sequence in $(X, \tau)$.

\section{Existence of basic sequences}

In this section we consider the question of whether an $F$-space need possess a basic sequence. The results we obtain will not be used in Section 5 , and this section may be omitted. We shall call a topological vector space $(E, \tau)$ minimal if for every Hausdorff vector topology $\rho \leqq \tau$ we have $\rho=\tau$. It is well known that $\omega$ is minimal if we restrict to locally convex topologies.

Proposition 4.1. $\omega$ is a minimal F-space.

Proof. Suppose $\rho$ is a weaker vector topology on $\omega$ and $x_{a} \rightarrow 0(\rho)$ but $\left\|x_{a}\right\| \geqq \theta$ (where $\|$.$\| is an F$-norm determining the topology of $\omega$ ). Then there is a sequence $\left(z_{n}\right)$, with $\left\|z_{n}\right\| \geqq \theta$, which is a basic sequence for some weaker Hausdorff vector topology on $\omega$ (Proof of 3.4). Let $E$ be the closed linear span of $\left(z_{n}\right)$ in the original topology, then $E \cong \omega$. However, the dual functionals of $\left(\mathrm{z}_{n}\right)$ induce on $E$ a weaker Hausdorff locally convex topology. It follows that $z_{n} \rightarrow 0$ contrary to assumption.

We do not know any other examples of minimal $F$-spaces; their existence is crucial to the problem of basic sequences in view of the following theorem.

Theorem 4.2. Every non-minimal F-space contains a basic sequence.

Before proceeding to the proof of Theorem 4.2 we first prove a stability theorem for basic sequences similar to a locally convex version given by Weill (13) (cf. also Shapiro (11), p. 1085). A sequence in a topological vector space is regular if it is bounded away from zero. 
Lemma 4.3. Suppose $X$ is an $F$-space and $\left(x_{n}\right)$ is a regular basic sequence. Suppose $\Sigma\left\|u_{n}\right\|<\infty$, and let $y_{n}=x_{n}+u_{n}$. If whenever

$$
\sum_{n=1}^{\infty} t_{n} y_{n}=0
$$

then $t_{n}=0$, then $\left(y_{n}\right)$ is also a basic sequence.

Proof. Define a map $S: l_{\infty} \rightarrow X$ by

$$
S(t)=\sum_{n=1}^{\infty} t_{n} u_{n}
$$

Since $\Sigma\left\|u_{n}\right\|<\infty, S$ is well defined and $S$ is continuous by the BanachSteinhaus Theorem. Now suppose $\left(t^{(n)}\right)$ is a sequence in $l_{\infty}$ such that

and

$$
\sup \left\|t^{(n)}\right\|_{\infty}<\infty
$$

$$
\lim _{n \rightarrow \infty} t_{k}^{(n)}=0 \text { for each } k .
$$

Then it is easy to verify that $\left\|S\left(t^{(n)}\right)\right\| \rightarrow 0$.

Let $E$ be the closed linear span of $\left\{x_{n}\right\}$ and suppose $f_{n} \in E^{\prime}$ is the bi-orthogonal sequence. For $x \in E, \lim _{n \rightarrow \infty} f_{n}(x)=0$, since $\left(x_{n}\right)$ is regular. We define $R: E \rightarrow c_{0}$ by $R(x)=\left(f_{n}(x)\right) ; R$ is continuous by the Closed Graph Theorem. Hence the map $T: E \rightarrow X$ defined by $T=I+S R$ is also continuous. Since $T$ takes the form

$$
T(x)=\sum_{n=1}^{\infty} f_{n}(x) y_{n} .
$$

$T$ is injective. Now suppose $\left(z_{n}\right) \subset E$ is a sequence such that $\left\|T\left(z_{n}\right)\right\| \rightarrow 0$; suppose $\left\|z_{n}\right\|>\varepsilon>0$. We suppose at first

$$
\sup _{n}\left\|R\left(z_{n}\right)\right\|_{\infty}<\infty .
$$

Then by selecting a subsequence we may suppose $R\left(z_{n}\right) \rightarrow t$ co-ordinatewise in $l_{\infty}$ and hence

Now

$$
S\left(R\left(z_{n}\right)\right) \rightarrow S(t) \text { in } X .
$$

Therefore $S(t) \in E$ and

$$
z_{n}=T\left(z_{n}\right)-S\left(R\left(z_{n}\right)\right) \rightarrow-S(t)
$$

i.e.

$$
R\left(z_{n}\right)+R S(t) \rightarrow 0 \text { in } l_{\infty} \text {. }
$$

and so

$$
\begin{aligned}
t+R S(t) & =0 \\
S(t)+S R S(t) & =0 \\
T(S(t)) & =0 \\
S(t) & =0
\end{aligned}
$$

$$
\lim _{n \rightarrow \infty} z_{n}=0
$$


contrary to assumption. It follows that no subsequence of $\left(\left\|R z_{n}\right\|_{\infty}\right)$ is bounded.

If, on the contrary, $\left\|R z_{n}\right\|_{\infty} \rightarrow \infty$, then we may consider $\left(\left\|R z_{n}\right\|_{\infty}^{-1} z_{n}\right)$ and obtain a similar contradiction. We establish that for such a sequence $\left\|R z_{n}\right\|_{\infty}^{-1} z_{n} \rightarrow 0$ and hence $\left\|R z_{n}\right\|_{\infty}^{-1} R z_{n} \rightarrow 0$ in $l_{\infty}$ which is a contradiction. Hence $T$ is an isomorphism on to its image, and as $T x_{n}=y_{n},\left(y_{n}\right)$ is a basic sequence.

Proof of Theorem 4.2. Let $U_{n}$ be a base of neighbourhoods of 0 in $(X, \tau)$; We may assume, without loss of generality, that $U_{1}$ is not a neighbourhood of 0 in some weaker vector topology. By Proposition 2.2 there are vector topologies $\alpha, \beta$ in $X$ such that $\alpha<\beta \leqq \tau, \beta$ is metrisable and $\alpha$-polar and $U_{1}$ is a $\beta$-neighbourhood. Then by Theorem 3.2 there is a basic sequence $\left(w_{k}^{(1)}\right)$ in $(X, \beta)$. Then let $E_{1}$ be the $\tau$-closed linear hull of the sequence $\left(w_{k}^{(1)}\right)$ and let $F_{1}$ be the linear span; let $\gamma_{1}=\beta$. Then by induction we construct sequences $\left(h_{k}^{(n)}\right), E_{n}$, $F_{n}, \gamma_{n}$ such that $F_{n}=\operatorname{lin}\left\{w_{k}^{(n)}: k=1,2, \ldots\right\}, E_{n}$ is the $\tau$-closure of $F_{n}$ and $\gamma_{n}$ is a metrisable vector topology on $E_{n}$ such that $\left(w_{k}^{(n)}: k=1,2, \ldots\right)$ is a basis of $\left(E_{n}, \gamma_{n}\right)$. Furthermore

(i) $\left(w_{k}^{(n)}\right)$ is block basic with respect to $\left(w_{k}^{(n-1)}\right)$ for $n \geqq 2$, i.e. $w_{k}^{(n)}$ takes the form

$$
w_{k}^{(n)}=\sum_{p_{k-1}+1}^{p_{k}} c_{i} w_{i}^{(n-1)}
$$

where $p_{0}=0<p_{1}<p_{2} \ldots$. Thus $F_{n} \subset F_{n-1}$ for $n \geqq 2$ and $E_{n} \subset E_{n-1} n \geqq 2$.

(ii) The topology $\gamma_{n}$ on $E_{n}$ is finer than $\gamma_{n-1}$ restricted to $E_{n}$ for $n \geqq 2$, and coarser than $\tau$.

(iii) $U_{n} \cap E_{n}$ is a $\gamma_{n}$-neighbourhood of 0 .

We now describe the inductive construction; suppose $\left(w_{k}^{(n)}\right), E_{n}, F_{n}$ and $\gamma_{n}$ have been chosen. If $U_{n+1} \cap E_{n}$ is a $\gamma_{n}$-neighbourhood of 0 then let $\gamma_{n+1}=\gamma_{n}$ and $w_{k}^{(n+1)}=w_{k}^{(n)}$ for all $k$. Otherwise by Proposition 2.2 we may find topologies $\alpha$ and $\gamma_{n+1}$ on $E_{n}$ such that $\gamma_{n} \leqq \alpha<\gamma_{n+1} \leqq \tau, \gamma_{n+1}$ is $\alpha$-polar and metrisable and $U_{n+1} \cap E_{n}$ is a $\gamma_{n+1}$-neighbourhood of 0 but not an $\alpha$-neighbourhood.

Since $F_{n}$ is $\tau$-dense in $E_{n}, F_{n}$ is also $\gamma_{n+1}$-dense and hence $\alpha<\gamma_{n+1}$ on $F_{n}$. Thus by Corollary 3.3 we may determine a $\gamma_{n+1}$-regular basic sequence $\left(z_{k}\right)$ in $F_{n}$ such that $z_{k} \rightarrow 0\left(\gamma_{n}\right)$. Thus

$$
z_{k}=\sum_{i=1}^{q(k)} c_{k, i} w_{i}^{(n)}
$$

where $\lim _{k \rightarrow \infty} c_{k, i}=0$ for each $i$ (since the co-ordinate functionals for $\left(w_{i}^{(n)}\right)$ are $\gamma_{n}$-continuous). It follows easily that we may find a subsequence $\left(y_{k}\right)$ and a block basic sequence $\left(w_{k}^{(n+1)}\right)$ such that $\sum_{k}\left\|y_{k}-w_{k}^{(n+1)}\right\|_{n+1}<\infty$ where $\|\cdot\|_{n+1}$ is an $F$-norm determining $\gamma_{n+1}$. If

$$
\sum_{k=1}^{\infty} t_{k} w_{k}^{(n+1)}=0 \quad\left(\gamma_{n+1}\right)
$$


then

$$
\sum_{k=1}^{\infty} t_{k} w_{k}^{(n+1)}=0 \quad\left(\gamma_{n}\right)
$$

and thus since the co-ordinate functionals for $w_{i}^{(n)}$ are $\gamma_{n}$-continuous $t_{k}=0$ for all $k$. Thus $\left(w_{k}^{(n+1)}\right)$ is a $\gamma_{n+1}$-basic sequence, and we proceed by letting $F_{n+1}=\operatorname{lin}\left\{w_{k}^{(n)}\right\}, E_{n+1}=\bar{F}_{n+1}$ (in $\tau$ ). This completes the inductive construction.

Finally take the "diagonal sequence"

$$
v_{n}=w_{n}^{(n)} \text {. }
$$

Then for each $n,\left(v_{k}: k \geqq n\right)$ is block basic with respect to $\left(w_{k}^{(n)}\right)$. In particular $\left(v_{k}\right)$ is block basic with respect to $\left(w_{k}^{(1)}\right)$ and hence there are $\gamma_{1}$-continuous linear functionals $\left(f_{k}\right)$ defined on lin $\left\{v_{k}\right\}$ such that $f_{i}\left(v_{j}\right)=\delta_{i j}$. These are then also $\tau$-continuous and extend to the closed linear span $H$ of $\left\{v_{k}\right\}$. Now suppose $x \in H$; we show

$$
\sum_{i=1}^{\infty} f_{i}(x) v_{i}=x
$$

For any $n,\left(v_{k}: k \geqq n\right)$ is a basic sequence in $\left(E_{n}, \gamma_{n}\right)$; let

$$
R_{n}(x)=x-\sum_{i=1}^{n-1} f_{i}(x) v_{i}
$$

Then $R_{n}(x)$ is in the $\tau$-closure of $\operatorname{lin}\left\{v_{k}: k \geqq n\right\}$, as this space is easily seen to be $\bigcap_{i=1}^{n-1} f_{i}^{-1}(0)$. Thus $R_{n}(x)$ is in $E_{n}$ and in the $\gamma_{n}$-closure of lin $\left\{v_{k}: k \geqq n\right\}$. Therefore

$$
R_{n}(x)=\sum_{i=n}^{\infty} f_{i}(x) v_{i} \quad\left(\gamma_{n}\right)
$$

and so for some $N$ and all $m \geqq N$,

and

$$
R_{n}(x)-\sum_{i=n}^{m} f_{i}(x) v_{i} \in U_{n}
$$

$$
x-\sum_{i=1}^{m} f_{i}(x) v_{i} \in U_{n} .
$$

Thus $x=\sum_{i=1}^{\infty} f_{i}(x) v_{i}$ for $x \in H$, and $\left(v_{i}\right)$ is a basic sequence.

If $E$ is a minimal $F$-space, then $E$ may still possess a basic sequence (see Proposition 4.1). The author does not know if every $F$-space must possess a basic sequence.

Theorem 4.4. Let $(X, \tau)$ be an F-space; the following are equivalent:

(i) $X$ contains no basic sequence.

(ii) Every closed subspace of $X$ with a separating dual is finite-dimensional. 
Proof. Clearly (ii) $\Rightarrow$ (i) so we have to show (i) $\Rightarrow$ (ii). If $E$ is a subspace of $X$ with a separating dual, then the weak topology $\sigma$ on $E$ is weaker than $\tau$. If $E$ is infinite-dimensional, then by Theorem $4.2 \sigma=\tau$. But in this case $E \cong \omega$, and so has a basis. Therefore, $E$ is finite-dimensional.

\section{Applications}

We now can apply basic sequences or rather semi-basic sequences to derive many results familiar in locally convex theory.

\section{Theorem 5.1.}

(i) Let $(X, \tau)$ be an F-space and suppose $\rho \leqq \tau$ is a vector topology on $X$ compatible with $\tau$. Then every $\rho$-bounded set is $\tau$-bounded.

(ii) Suppose $X$ is a vector space and $\rho \leqq \tau$ are two vector topologies on $X$ such that $\rho$ and $\tau$ are compatible and $\tau$ is $\rho$-polar. Then any $\rho$-bounded set is $\tau$-bounded.

Proof. (i) It is enough to show that if $x_{n} \rightarrow 0(\rho)$ and $c_{n}$ is a sequence of scalars such that $c_{n} \rightarrow 0$ then $c_{n} x_{n} \rightarrow 0(\tau)$. Suppose $x_{n} \rightarrow 0(\rho)$; then choose $x_{0} \neq 0$. For $c_{n} \rightarrow 0, c_{n} \neq 0$,

$$
c_{n}\left(x_{n}+x_{0}\right) \rightarrow 0(\rho) \text {. }
$$

Suppose $c_{n}\left(x_{n}+x_{0}\right) \rightarrow 0(\tau)$; then by Corollary 3.4 , there is a semi-basic sequence $\left(z_{n}\right)$ with $z_{1}=x_{0}$ and

$$
z_{n}=c_{m_{n}}\left(x_{m_{n}}+x_{0}\right) \quad(n \geqq 2),
$$

where $\left(m_{n}\right)$ is an increasing sequence of integers. Then

$$
c_{m_{n}}^{-1} z_{n} \rightarrow x_{0}(\rho)
$$

and hence $x_{0}$ is in the $\rho$-closure of $\operatorname{lin}\left\{z_{n}: n \geqq 2\right\}$. Thus $x_{0}$ is also in the $\tau$-closure of $\operatorname{lin}\left\{z_{n}: n \geqq 2\right\}$, contradicting the fact that $\left(z_{n}\right)$ is a semi-basic sequence. Thus since $c_{n} x_{0} \rightarrow 0, c_{n} x_{n} \rightarrow 0(\tau)$.

The proof of (ii) is somewhat similar; let $\eta$ be a $\rho$-lower-semi-continuous $\tau$-continuous $F$-semi-norm and let $N=\{x: \eta(x)=0\}$. Then $X / N$ is metrisable under $\eta$ and may be given the quotient topology $\hat{\rho}$ of $\rho$ ( $N$ is $\rho$-closed). Every $\eta$-closed subspace of $X / N$ is $\hat{\rho}$-closed and so an argument similar to (i) may be employed.

Corollary 5.2. Suppose $(X, \tau)$ is an $F$-space and $\rho \leqq \tau$ is a metrisable vector topology compatible with $\tau$. Then $\rho=\tau$.

Corollary 5.3. Let $(X, \tau)$ be an F-space with the Hahn-Banach Extension Property. Then $X$ is locally convex.

Proof. Let $\sigma$ be the weak topology on $N$; then $\sigma \leqq \tau$ and $\sigma$ and $\tau$ are compatible by the HBEP. For suppose $Y$ is a $\tau$-closed subspace and $x \notin Y$; then 
by HBEP there is a continuous linear functional $\phi$ such that $\phi(Y)=0$ and $\phi(x)=1$. Let $\mu$ be the associated Mackey topology; then (see Shapiro (10), Proposition 3) $\sigma \leqq \mu \leqq \tau$ and $\mu$ is metrisable. Hence by Corollary $5.2 \mu=\tau$ and $\tau$ is locally convex.

Corollary 5.4. Suppose $(X, \tau)$ is an F-space and $\rho \leqq \tau$ is a vector topology compatible with $\tau$. Then $\tau$ is $\rho$-polar.

Proof. Let $\gamma$ be the topology induced by the $\rho$-closures of $\tau$-neighbourhoods of 0 ; then $\rho \leqq \gamma \leqq \tau$ and $\gamma$ is metrisable. Hence by 5.2, $\gamma=\tau$.

Theorem 5.5. Let $(X, \tau)$ be an F-space and let $\left(x_{n}\right)$ be a basis of $X$ in a compatible topology $\rho \leqq \tau$. Then $\left(x_{n}\right)$ is a basis of $X$.

Proof. By the previous corollary we may assume that $\tau$ is defined by a $\rho$-lower-semi-continuous $F$-norm $\|$.$\| (see Proposition 2.1). Each x \in X$ may be expanded in the form

$$
x=\sum_{i=1}^{\infty} f_{i}(x) x_{i}(\rho)
$$

(the linear functionals $f_{n}$ are not necessarily $\rho$-continuous). Now for each $x \in X$, the sequence $\left(\sum_{i=1}^{n} f_{i}(x) x_{i}\right)$ is $\rho$ - and therefore $\tau$-bounded (Theorem 5.1) and so we may define

$$
\|x\|^{*}=\sup _{n}\left\|\sum_{i=1}^{n} f_{i}(x) x_{i}\right\| .
$$

Then $\lim \|t x\|^{*}=0$ since $\lim t y=0$ uniformly for $y$ in a bounded set; hence $\|\cdot\|^{*} \stackrel{t \rightarrow 0}{\text { is }}$ an $F$-norm on $X$. Clearly also $\|x\|^{*} \geqq\|x\|$ by the $\rho$-lower-semicontinuity of $\|\cdot\|$.

It remains to establish that $\left(X,\|\cdot\|^{*}\right)$ is complete and then by the Closed Graph Theorem it will follow that $\|\cdot\|^{*}$ and $\|$.$\| are equivalent. Let \left(y_{n}\right)$ be a $\|\cdot\|^{*}$-Cauchy sequence; then since $\left\|y_{n}-y_{m}\right\| \leqq\left\|y_{n}-y_{m}\right\|^{*}$ for all $m, n,\left(y_{n}\right)$ is $\tau$-convergent to $y$ say. Furthermore, it can be seen that the sequences

$$
\left(\sum_{i=1}^{m} f_{i}\left(y_{n}\right) x_{i}\right)
$$

are $\tau$-convergent uniformly in $m$; clearly $\lim _{n \rightarrow \infty} f_{i}\left(y_{n}\right)=t_{i}$ exists and

$$
\lim _{n \rightarrow \infty} \sum_{i=1}^{m} f_{i}\left(y_{n}\right) x_{i}=\sum_{i=1}^{m} t_{i} x_{i}
$$

uniformly in $m$ for the topology $\tau$. Thus working in the weaker topology $\rho$

$$
\lim _{m \rightarrow \infty} \sum_{i=1}^{m} t_{i} x_{i}=\lim _{n \rightarrow \infty} \lim _{m \rightarrow \infty} \sum_{i=1}^{m} f_{i}\left(y_{n}\right) x_{i}=y .
$$


(The limits are interchangeable by uniform convergence.) Therefore it follows that

$$
\lim _{n \rightarrow \infty} \sum_{i=1}^{m} f_{i}\left(y_{n}\right) x_{i}=\sum_{i=1}^{m} f_{i}(y) x_{i}(\tau)
$$

uniformly in $m$ and that $\left\|y-y_{n}\right\|^{*} \rightarrow 0$. Hence $\|\cdot\|$ and $\|\cdot\|^{*}$ are equivalent, and by an application of Lemma $2.4,\left(x_{n}\right)$ is a basic sequence in $(X,\|\cdot\|)$. By the compatibility of $\rho,\left(x_{n}\right)$ is a basis of $X$.

Shapiro (12) proves that the Weak Basis Theorem fails in any non-locally convex locally bounded $F$-space. With regard to this theorem we establish that a weaker version of the Weak Basis Theorem holds always.

Proposition 5.6. Let $\left(x_{n}\right)$ be a weak basis of $(X, \tau)$, where $(X, \tau)$ is an F-space with a separating dual. Then the associated linear functionals $\left\{f_{n}\right\}$ are continuous.

Proof. Let $\sigma$ be the weak topology and $\mu$ the (metrisable) Mackey topology. Then $(X, \mu)$ is barrelled, for if $C$ is a $\mu$-barrel then $C$ is $\tau$-closed and by the Baire Category Theorem we may show $C$ has $\tau$-interior. It follows easily that $C$ is a $\tau$-neighbourhood of 0 and thus a $\mu$-neighbourhood ((10), Proposition 3).

Now let $\|\cdot\|_{n}$ be a sequence of semi-norms defining $\mu$ and let

$$
\|x\|_{n}^{*}=\sup _{m}\left\|\sum_{i=1}^{m} f_{i}(x) x_{i}\right\|_{n}
$$

(finite, since $\mu$ and $\sigma$ have the same bounded sets). Let $\mu^{*}$ be the topology induced by the sequence $\|\cdot\|_{n}^{*}$ and let $\hat{X}$ be the $\mu^{*}$-completion of $X$. Consider the identity map $i:(X, \mu) \rightarrow\left(\hat{X}, \mu^{*}\right)$. Suppose $z_{n} \in X, z_{n} \rightarrow z(\mu)$ and $z_{n} \rightarrow z^{\prime}\left(\mu^{*}\right)$. Then $\left\{\sum_{i=1}^{m} f_{i}\left(z_{n}\right) x_{i}\right\}_{n=1}^{\infty}$ is uniformly $\mu$-Cauchy for $m=1,2, \ldots$; thus in the topology $\sigma \leqq \mu$

$$
\lim _{n \rightarrow \infty} \lim _{m \rightarrow \infty} \sum_{i=1}^{m} f_{i}\left(z_{n}\right) x_{i}=\lim _{m \rightarrow \infty} \lim _{n \rightarrow \infty} \sum_{i=1}^{m} f_{i}\left(z_{n}\right) x_{i}
$$

and we conclude

and

$$
\lim _{n \rightarrow \infty} f_{i}\left(z_{n}\right)=t_{i} \text { exists for each } i
$$

$$
\lim _{n \rightarrow \infty} z_{n}=z=\sum_{i=1}^{\infty} t_{i} x_{i} \text { in } \sigma .
$$

Thus $f_{i}(z)=t_{i}$ and therefore

$$
\lim _{n \rightarrow \infty} \sum_{i=1}^{m} f_{i}\left(z_{n}-z\right) x_{i}=0 \mu \text {-uniformly in } m .
$$

Hence $z_{n} \rightarrow z$ in $\left(X, \mu^{*}\right)$ and $i$ has Closed Graph. By the Closed Graph Theorem ((9), p. 116), since $\left(\widehat{X}, \mu^{*}\right)$ is complete and metric, $\mu \geqq \mu^{*}$ and it follows easily that each $f_{n}$ is $\mu$ and hence $\tau$-continuous.

The idea of the next theorem is due to Pelczynski (7). 
Theorem 5.7. Let $(X, \tau)$ be an F-space and suppose $\rho \leqq \tau$ is a compatible vector topology. Let $K$ be a subset of $X$; then the following are equivalent

(i) $K$ is $\rho$-compact,

(ii) $K$ is $\rho$-sequentially compact,

(iii) $K$ is $\rho$-countably compact.

Proof. (i) $\Rightarrow$ (iii) and (ii) $\Rightarrow$ (iii) are well known. Let $\|\cdot\|$ be an $F$-norm determining $\tau$; by Corollary 5.4 we may suppose $\|$.$\| is \rho$-lower-semi-continuous.

(iii) $\Rightarrow$ (i). It is easy to see that $K$ is $\rho$-precompact; we show that $K$ is also $\rho$-complete. Let $(\hat{X}, \hat{\rho})$ be the $\rho$-completion of $X$ and let $Y \subset \hat{X}$ be the vector space of all $y \in \hat{X}$ such that there is a $\rho$-bounded net $x_{\alpha} \in X$ such that $x_{\alpha} \rightarrow y$. By Theorem 5.1 a $\rho$-bounded net is $\tau$-bounded. Let $B_{\lambda}=\{x \in X:\|x\| \geqq \lambda\}$; then for $y \in Y$ we define

$$
\|y\|^{*}=\inf \left\{\lambda: y \in \bar{B}_{\lambda} \text {, closure in } \hat{\rho}\right\} .
$$

Let $y \in Y$ and suppose $x_{\alpha}$ is a $\tau$-bounded net converging to $y$ in $\hat{\rho}$; then

and

$$
\|y\|^{*} \leqq \sup _{\alpha}\left\|x_{\alpha}\right\|<\infty
$$

$$
\begin{aligned}
\lim _{t \rightarrow 0}\|t y\|^{*} & \leqq \lim _{t \rightarrow 0} \sup _{\alpha}\left\|t x_{\alpha}\right\| \\
& =0
\end{aligned}
$$

since the net $\left\{x_{\alpha}\right\}$ is bounded (cf. Theorem 5.5). It follows without difficulty that $\|.\|^{*}$ is an $F$-semi-norm on $Y$, and that $\|.\|^{*}$ is $\hat{\rho}$-lower-semi-continuous; also from the definition, $\|x\|=\|x\|^{*}$ for $x \in X$, since each $B_{\lambda}$ is $\rho$-closed. Next if $y \in Y$ and $\|y\|^{*}=0$ then for each $\lambda>0$ and $V$ a neighbourhood of 0 in $(\hat{X}, \hat{\rho})$ we may find $x_{\lambda, V} \in X$ such that $x_{\lambda, V}-y \in V$ and $\left\|x_{\lambda, V}\right\| \leqq \lambda$. The set $\{(\lambda, V): \lambda>0, V$ a $\hat{\rho}$-neighbourhood of 0$\}$ is directed in the obvious way $\left[(\lambda, V) \geqq\left(\lambda^{\prime}, V^{\prime}\right)\right.$ if and only if $\lambda \leqq \lambda^{\prime}$ and $\left.V \subset V^{\prime}\right]$; then the net $x_{\lambda, V}$ converges to 0 in $(X, \tau)$ and $x_{\lambda, v} \rightarrow 0$ in $(X, \rho)$. However $x_{\lambda, V} \rightarrow y$ in $(\hat{X}, \hat{\rho})$ and so $y=0$ Thus $Y$ is a metrisable vector space under $\left\|^{*}\right\|^{*}$ and $\|\cdot\|^{*}$ is $\hat{\rho}$-lower-semicontinuous.

Now suppose $x_{\alpha} \in K$ is a $\rho$-Cauchy net; then $x_{\alpha} \rightarrow y$ in $(\hat{X}, \hat{\rho})$ and $y \in Y$. Suppose at first $\left\|x_{\alpha}-y\right\|^{*} \rightarrow 0$; then by the completeness of $(X, \tau) y \in X$, and there is a sequence $(\alpha(n))$ such that $x_{\alpha(n)} \rightarrow y(\tau)$. Thus $y$ is the sole $\rho$-cluster point of $\left\{x_{\alpha(n)}\right\}$ in $X$; since $K$ is countably compact, $y \in K$, and $x_{\alpha} \rightarrow y$ in $(K, \rho)$.

Now suppose $\left\|x_{\alpha}-y\right\|^{*} \rightarrow 0$ and that $y \notin X$; since $y \neq 0$ we may suppose $x_{\alpha} \notin V$ for all $\alpha$, where $V$ is a $\rho$-neighbourhood of 0 . Then by Theorem 3.2 there is a basic sequence $\left(z_{n}\right)$ in $\left(Y,\|\cdot\|^{*}\right)$ such that:

(i) $z_{1}=y$.

(ii) $z_{n}=w_{n}-y, n \geqq 2$ where $w_{n}=x_{\alpha(n)}$ for some increasing sequence.

(iii) inf $\left\|z_{n}\right\|^{*}>0$. 
Let $Z$ be the closed linear span of $\left\{z_{n}\right\}_{n=1}^{\infty}$ and let $W$ be the closed linear span of $\left\{w_{n}\right\}_{n=2}^{\infty}$. Since $z_{1} \notin X$ and $W \subset X, W$ is a closed subspace of co-dimension one in $Z$. Let $\phi$ be the continuous linear functional on $\left(Z,\|\cdot\|^{*}\right)$ such that $\phi\left(z_{1}\right)=1$ and $\phi(W)=0$; we define $A: Z \rightarrow Z$ by $A z=z-\phi(z) z_{1}$. Then for $n \geqq 2$

Similarly define $B: Z \rightarrow Z$ by

$$
\begin{aligned}
A z_{n} & =A w_{n}-A z_{1} \\
& =w_{n} .
\end{aligned}
$$

Then

$$
B\left(\sum_{i=1}^{\infty} t_{i} z_{i}\right)=\sum_{i=2}^{\infty} t_{i} z_{i}
$$

$$
\begin{aligned}
B w_{n} & =B\left(z_{1}+z_{n}\right) \\
& =z_{n} .
\end{aligned}
$$

It follows that $B A z_{n}=z_{n}, n \geqq 2$ and hence that $A$ is an isomorphism of ॉin $\left\{z_{n}: n \geqq 2\right\}$ on to its image. In particular $\left(w_{n}: n \geqq 2\right)$ is a basic sequence in $(X,\|\cdot\|)$. However $w_{n} \in K$ for $n \geqq 2$, and so $\left(w_{n}\right)$ possesses a $\rho$-cluster point. Now suppose $w_{0}$ is a $\rho$-cluster point; then $w_{0}$ is in the $\tau$-closed linear span of $\left(w_{n}\right)$ by compatibility. It follows that

$$
w_{0}=\sum_{i=2}^{\infty} \psi_{i}\left(w_{0}\right) w_{i}
$$

where $\psi_{i}$ is the dual sequence of $\tau$-continuous linear functionals on $W$. Each $\psi_{i}$ is also $\rho$-continuous by compatibility and hence

$$
\psi_{i}\left(w_{0}\right)=0 \quad i \geqq 2 .
$$

Therefore $w_{0}=0$. This contradicts the original choice of $x_{\alpha} \notin V$, where $V$ is a $\rho$-neighbourhood of 0 . Thus we have a contradiction.

Finally suppose $\left\|x_{\alpha}-y\right\|^{*} \rightarrow 0$ and $y \in X$; determine the basic sequence $\left(z_{n}: n \geqq 2\right)$ satisfying (ii)-(iii). In this case if $w_{0}$ is a $\rho$-cluster point of $\left(w_{n}: n \geqq 2\right)$ then $w_{0}-y$ is a $\rho$-cluster point of $\left(z_{n}: n \geqq 2\right)$. Since $w_{0}-y \in X$ and $z_{n} \in X$ we conclude that $w_{0}-y$ is in the $\tau$-closed linear span of $\left\{z_{n}: n \geqq 2\right\}$ by compatibility and it follows as usual that $w_{0}-y=0$. Hence $y \in K$. We conclude that any $\rho$-Cauchy net converges in $K$ and so $K$ is complete and therefore compact.

(iii) $\Rightarrow$ (ii). Let $\left(x_{n}\right)$ be a sequence in $K$ and let $x_{0}$ be a $\rho$-cluster point. Then there is a net $\left(z_{\alpha}\right)$ in $K$ such that each $z_{\alpha}$ is some $x_{n}$ and $z_{\alpha} \rightarrow x_{0}(\rho)$. If $z_{\alpha} \rightarrow x_{0}$ in $\tau$ then there is nothing to prove, as it will follow that some subsequence of $\left(x_{n}\right)$ converges to $x_{0}$. Otherwise we may find a basic sequence $\left(u_{n}\right)$ of the form $u_{n}=z_{\alpha(n)}-x_{0}$. Let $w$ be a $\rho$-cluster point of $\left(z_{\alpha(n)}\right)$ in $K$; then clearly $w-x_{0} \in \overline{\operatorname{lin}}\left\{u_{n}\right\}$ and since $\tau$ and $\rho$ are compatible it follows as in (iii) $\Rightarrow$ (i) that $w-x_{0}=0$. Hence $x_{0}$ is the sole cluster point of $\left(z_{\alpha(n)}\right)$ and so $z_{\alpha(n)} \rightarrow x_{0}$. However $z_{\alpha(n)}$ is simply a subsequence of $\left(x_{n}\right)\left(\alpha(n) \rightarrow \infty\right.$ since the $z_{\alpha(n)}$ are distinct). 
[ADDED IN PROOF: The problem of determining conditions under which the Hahn-Banach Extension Property is equivalent to local convexity was originally posed by Duren, Romberg and Shields (14) p.59.]

\section{REFERENCES}

(1) C. Bessaga and A. Pelczynski, Wlansosci baz $w$ przestrzeniach typu $B_{0}$, Prace Mat. 3 (1959), 123-142.

(2) C. Bessaga and A. Pelczynski, On bases and unconditional convergence of series in Banach spaces, Studia Math. 17 (1958), 151-164.

(3) M. M. DAy, On the basis problem in normed linear spaces, Proc. Amer. Math. Soc. 13 (1962), 655-658.

(4) B. Gelbaum, Notes on Banach spaces with bases, An. Acad. Brasil. Ci. 30 (1958), 29-36.

(5) J. L. Kelley, General topology (New York, 1955).

(6) A. Pelczynski, A note on the paper of I. Singer, "Basic sequences and reflexivity of Banach spaces", Studia Math. 21 (1962), 371-374.

(7) A. Pelczynski, A proof of the Eberlein-Smulian Theorem by an application of basic sequences, Bull. Acad. Polon. Sci. Sér. Math. Astronom. Phys. 12 (1964), 543548.

(8) M. RIBE, Necessary convexity conditions for the Hahn-Banach theorem in metrizable spaces, Pacific J. Math. 44 (1973), 715-732. 1964).

(9) A. P. Robertson and W. Robertson, Topological vector spaces (Cambridge,

(10) J. H. Shapiro, Extension of linear functionals on $F$-spaces with bases, Duke Math. J. 37 (1970), 639-645.

(11) J. H. SHAPIRo, On convexity and compactness in $F$-spaces with bases, Indiana Univ. Math. J. 21 (1972), 1073-1090.

(12) J. H. SHAPIRo, On the weak basis theorem in F-spaces (to appear).

(13) L. J. WeILL, Stability of bases in complete barrelled space, Proc. Amer. Math. Soc. 18 (1967), 1045-1050.

(14) P. L. Duren, B. W. Romburg and A. L. Sheidls, Linear functionals on $\mathbf{H}^{P}$-spaces with $0<p<1, J$. Reine Angew. Math. 238 (1969), 32-60.

\section{UNIVERSITY COLLEGE \\ Singleton PARK \\ SWANSEA SA2 8PP}

